\title{
Depth error concealment based on decision making
}

\begin{abstract}
One of the common form of representing stereoscopic video is combination of $2 \mathrm{D}$ video with its corresponding depth map which is made by a laser camera to illustrate depth in the video. When this type of video is transmitted over error prone channels, the packet loss leads to frame loss; and mostly this frame lost occur in depth frames. Thus, a depth error concealment based on decision making termed as DM-PV, which exploits high correlation of 2-D image and its corresponding depth map. The 2D image provide information about the missing frame in the depth sequence to assist the decision making process in order to conceal the lost frames. The process involves inserting proper blank frame and duplication of previous frames instead of missing frames in depth sequence. PSNR performance improves over frame copy method has no decision making. Furthermore, subjective quality of stereoscopic video is better using DM-PV.
\end{abstract}

